\title{
A SIMPLE SYSTEM FOR THIN ROD BENDING MEASUREMENT
}

\author{
Jan Hošek, Šárka Němcová \\ Faculty of Mechanical Engineering, Czech Technical University in Prague, \\ Technická 4, Prague 6, Czech Republic \\ jan.hosek@fs.cvut.cz
}

\begin{abstract}
A system for load and deformation measurement of small tubular samples is pre-sented. The system allows for a loading force measurement with a strain gauge load cell, a deformation measurement with an optical triangulation sensor and a needle deformation measurement with a remote camera. The paper presents the system structural design, the system calibration and its application for the needle bending test measurement. The system was used for the measurement of an eye endoscope prototype.
\end{abstract}

Keywords: Load cell, Rod, Bending, Deformation, Endoscope, Eye Surgery.

\section{Introduction}

Mo For the design of a probe of an eye endoscope, the trade-off between the mechanical dimensions and the rigidity is crucial. The target is the smallest wall thickness possible and there the accuracy of the simulations must be proven by an experiment. Stress and strain measurement is a common technique for testing mechanical structures. There exists a large variety of bend loading machines for testing standard samples. Such universal systems are typically large so as to achieve sufficient stiffness for reliable data measurement. Small or portable systems are usually custom designed to meet demands for special sample measurement. In our case we aim for a small portable loading system capable to produce a desired level of loading force $(0-10) \mathrm{N}$ in a small contact point and able to measure an induced sample deformation caused by the load. The system needs to be rigid and clearance-free to give reliable data measurement.

We want to use such a system for loading tests of various samples, but firstly, we want to use it for stiffness measurement of a needle-like tubular structure to simulate mechanical conditions and loads on a miniature endoscope under eye surgery operation. A standard commercial testing systems are designed to follow the requirements of ISO 80369 standards. We also found few examples of needle loading systems designed for different aims, usually to test a needle bending during its insertion to a tissue [1, 2, 3]. Eventually, we designed a new system which differs from the standard design of loading systems, but which fulfilled our needs.

This paper presents the system structural design, the system calibration and its application for the needle bending test measurement.

\section{Loading System Design}

The system design must solve few possible problems. While the needle is a relatively flexible structure we have expected a relatively large deformation under a small force load. For this reason, there is needed to solve how to separate the sample deformation measurement from the loading structure motion position measurement.

The loading structure needs to be provided with an exact contact point to the sample. The applied force must be measured together with the deformation of a commercial load cell provided with strain gauges. While the load cell is deformed by the applied force, the sample deformation needs to be measured as a position change of a mechanical part in front of the load cell to eliminate the influence of the load cell deformation to the measured data. The needle deformation can be measured e.g. with a camera. To achieve even higher resolution, we decided to measure the position of a stiff part of the loading cell directly, with a precise optical gauge. In such a case the actual position of the basement part of the load cell is not important. The only demand on the basement part of the load cell is that its motion must be clearance-free. Such motion is commonly provided with linear guides and a driving lead screw, at least two or three precise and expensive parts. As we expect the sample deformations in a range of few millimeters we replaced the common linear motion with a rotary motion around a remote center of motion. An approximate motion of this kind can be easily realized as clearance-free with a flexure rotary joint or a self-aligning ball support.

A measured sample is fixed in an XYZ movable support where no fine motions are needed. 
The fine loading motion is performed just by the loading system rotation. Keeping in mind the Abbe principle [4], we needed to keep the loading axis free for the placement of a distance measurement gauge. This condition leads to the use of a bar cell gauge allowing for an off axis design of the system. We used a commercially available $1 \mathrm{~kg}$ bar load cell 775-018 GM ELECTRONIC with the HX711 balance module, processed by an Arduino Uno minicomputer.

To achieve a point-like load contact to a tubular needle we provided the load cell with a contact edge perpendicular to the sample axis. The edge was realized by a right angle optical prism. The contact between the prism and the needle surface occurs at the prism bevel surface or its edge during the loading. The prism fixed to the load cell is always loaded with compression force only.

The basement of the load cell is an aluminum beam allowing for a rotary motion around the axis at the distance of $L=150 \mathrm{~mm}$ from the applied load force contact position. The rotary axis is formed by centers of two balls in self-aligning ball supports of diameter $D=6 \mathrm{~mm}$. A small axial preload eliminates any clearance. The mass and the corresponding torque of the aluminum beam is fully compensated for with a soft compression spring. From this zero position the aluminum beam with the load cell can be adjusted with a micrometer screw in contact with a ball fixed on the top of the beam. The real position of the load cell part in contact with the sample is measured with the Micro-Epsilon optoNCDT 1402-5 gauge having $0.6 \mu \mathrm{m}$ static distance resolution in 5 $\mathrm{mm}$ wide position measurement working range, and under $1.5 \mathrm{kHz}$ reading frequency. The overall view to the physical realization of the loading system with manual load adjustment and electronic loading force and contact position reading is shown in Fig 1.

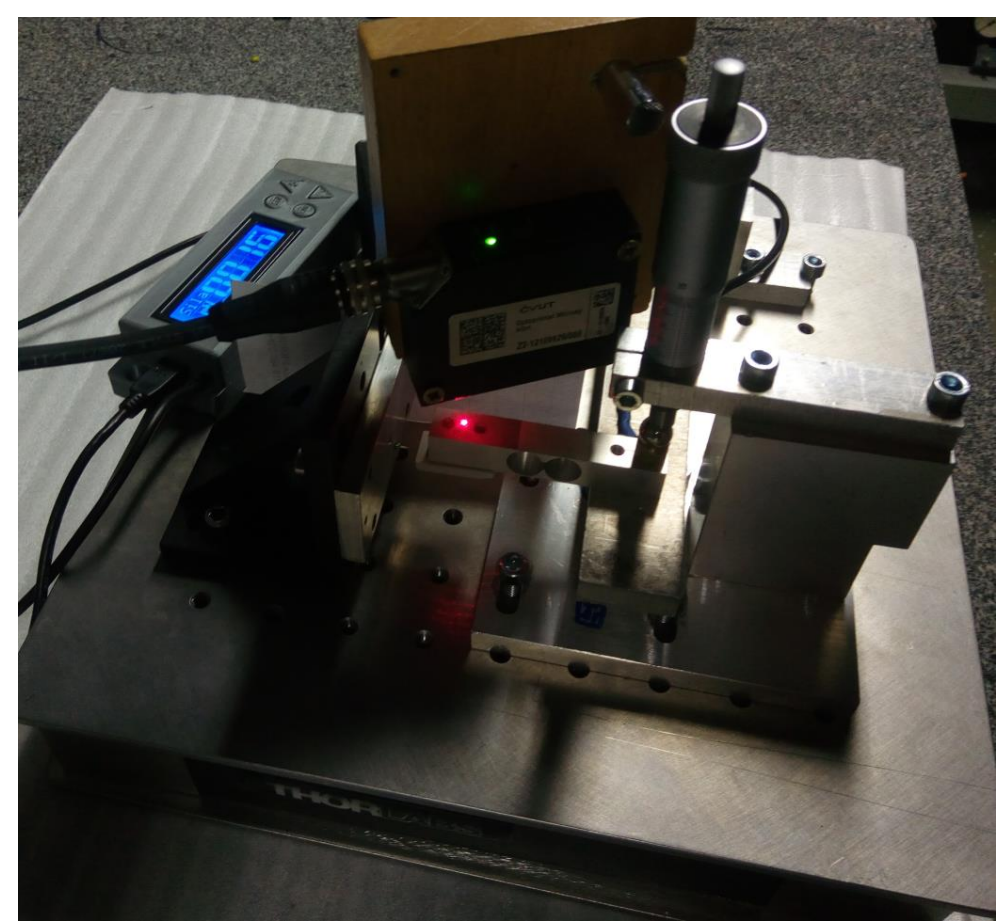

Figure 1: Physical realization of compact loading system with manual load adjustment and electronic loading force and sample contact position reading.

\section{Loading System Analysis}

The system load cell is permanently fixed to the aluminum beam so they move together by rotation around rotational axis made by two balls in contact with precisely reamed holes and lifted up with a soft spring so as to be in a point contact with the micrometer screw contact surface. The load cell can be assumed as three independently movable parts connected together with clearance-free flexure rotary joints (Fig. 2). The Part 3 of the load cell is permanently fixed to the aluminum beam and performs the same rotary motion around the axis as the aluminum beam.
The Part 2 consists of a parallelogram composed of two levers rotating in four flexure rotary joints. The Part 1 represents a rigid metal beam of the load cell capable to perform a linear motion with regard to the Part 3 when a non-zero force $F$ is applied to the loading edge of the optical prism. This position change of the Part 3 is registered with the noncontact optical sensor magnetically fixed to the loading system basement. It provides the Part 3 position information independently on whether it was moved by micrometer screw adjustment or the load cell response to the contact force $F$. A detail view of the load cell and a schematic view of its Parts 1, 2, and 3 is shown in Fig 2. 

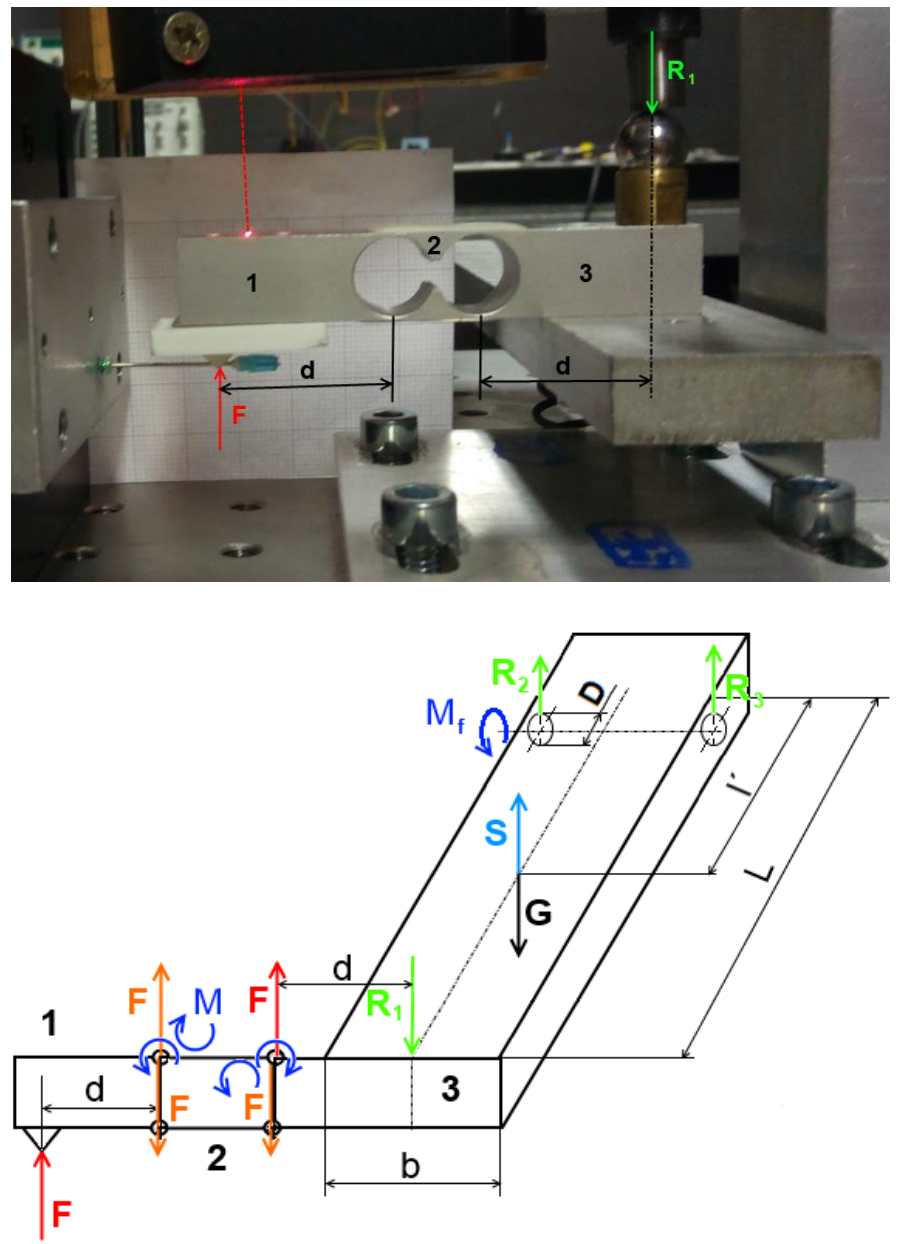

Figure 2: A detail view to the load cell and the schematic view to its Parts 1, 2 and 3 with all forces and torques emerging within the system.

The force $F$ applied to the Part 1 is compensated for with the inverse reaction $F$ and torque $M$ at the contact with the Part 2. The Part 2 transfers the reaction and toque to the contact with the Part 3, where the force $F$ emerges as the same as the contact force $F$. The torque $M$ is given by relation

$$
M=F d \text {. }
$$

The Part 3 must reach a static balance described by equations

$$
\begin{aligned}
& M+F d+R_{2} \frac{b}{2}-R_{a} \frac{b}{2}=0 \\
& F-R_{1}+S-G+R_{a}+R_{2}=0 \\
& F L-R_{1} L+(S-G) I-M_{f}=0,
\end{aligned}
$$

where $b=30 \mathrm{~mm}$ is the ball support width, $d=26.5$ $\mathrm{m}$ is the distance from the flexure joint to the force axis. $G$ is the gravity force compensated by the spring force $S=G+k \varphi l^{\prime}$, where $k$ it the spring stiffness, $\varphi$ is the rotation angle around the axis measured from the balance position where $S=G, I^{\prime}=100 \mathrm{~mm}$ and $L=$ $150 \mathrm{~mm}$ are the distances of the corresponding forces from the rotational axis, and $M_{f}$ is the friction torque given by relation:

$$
M_{f}=f \frac{D}{2}\left(R_{a}+R_{2}\right)
$$

The reactions $R_{2}$ and $R_{3}$ compensate the torque produced by the contact force $F$. The reactions can be expressed by relations:

$$
\begin{aligned}
& R_{\mathrm{a}}=R_{0}+R^{*} \\
& R_{2}=R_{0}-R^{*},
\end{aligned}
$$

By adding and subtracting the formulae (6) and (7) we get:

$$
\begin{aligned}
& R_{a}+R_{2}=2 R_{0} \\
& R_{a}-R_{2}=2 R^{\prime} .
\end{aligned}
$$

Solving for the individual reactions we get:

$$
\begin{aligned}
& R^{x}=2 \frac{d}{b} F=1.766 F
\end{aligned}
$$

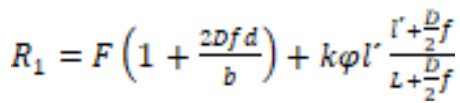

$$
\begin{aligned}
& R_{0}=F\left(\frac{1}{2}+\frac{D f d}{b}\right)+\frac{k}{2} \varphi I^{r}\left(\frac{T^{n}+\frac{D}{2} f}{L+\frac{B}{2} f}-1\right) .
\end{aligned}
$$


The loading system design dimensions were selected to reach a high ratio of the friction torque $\operatorname{arm} D / 2$ and the actuation torque $\operatorname{arm} L$, where $2 L / D$ $=50$. This assures that the system does not possess any motion backlash due to the friction. We also analyzed the influence of the approximate motion of the contact edge to the applied force $F$. The force $F$ is changed by $10^{-4}$ times for the $z$ travel distance up to $\pm 2.12 \mathrm{~mm}$ from the horizontal position. If there is acceptable force $F$ uncertainty of $10^{-3} F$, the total travel in $z$ axis around the horizontal position can reach up to $\pm 6.71 \mathrm{~mm}$, which is much more than the used optical distance sensor working range.

\section{The Needle Deformation Measurement}

The aim of the system is to use it for various loading reasons. Actually we are interested in bending characteristics of an eye endoscope under development. We decided to simulate the eye endoscope bending with a bending of a syringe needle of corresponding dimension 23G (i.e. outer diameter $0.635 \mathrm{~mm}$ ).

\section{a. Needle Three-Point Bending Test}

The 3-point bending test is a standardized test method for determination of flexural properties of beam-like specimens. For this reason, we used it as the first insight to the experimental task of the needle bending.

We put the needle on top of two $9 \mathrm{~mm}$ diameter rollers with center's distance of $19 \mathrm{~mm}$. We adjusted the contact loading to the center of the rollers and performed the loading in $100 \mu \mathrm{m}$ steps set with the micrometer screw.

The needle deformation response is shown in Figure 3.

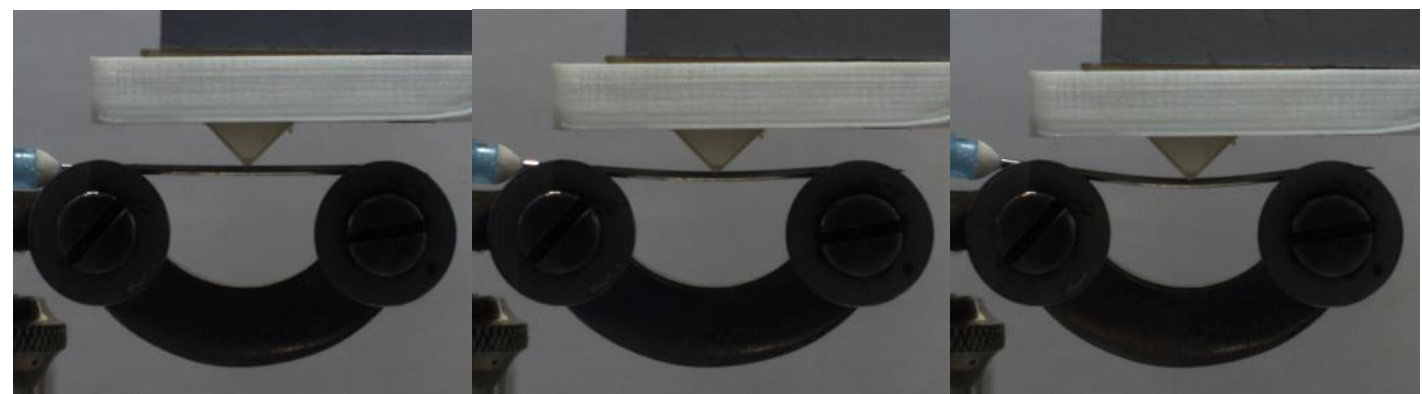

Figure 3: A detail view to the tested needle under load from zero (left image) to maximum used load (right image).

We took simultaneous reading of the load cell signal with $3.5 \mathrm{~Hz}$ and distance gauge signal with $50 \mathrm{~Hz}$ frequency. We synchronized the data to the maximum load time point and interpolated them to

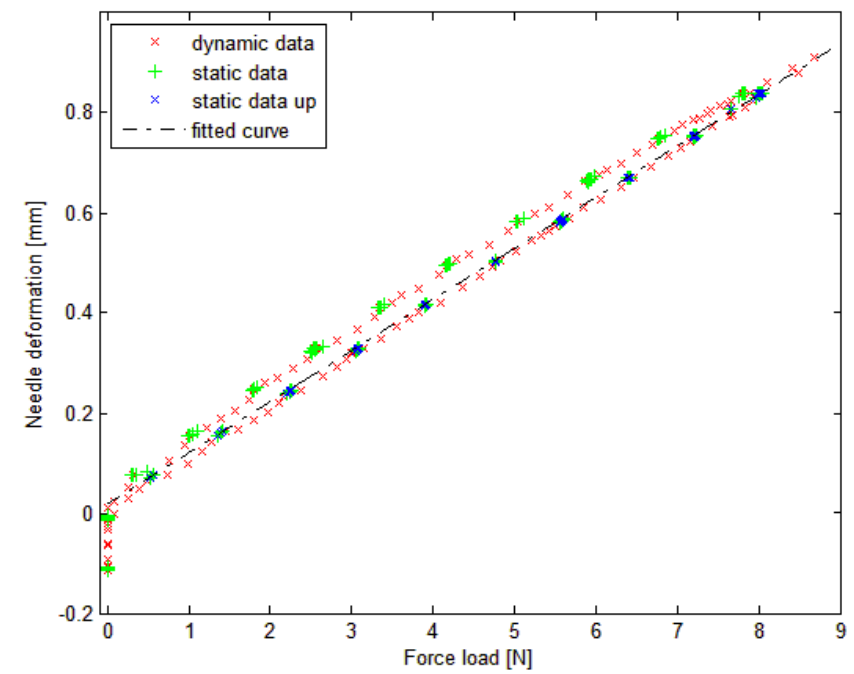

Figure 4: The needle deformation measurement data under 3-point bending test.

The measured data show different characteristics for individual set position values by the micrometer screw and for dynamic motion in between those receive a limited number of data for further processing.

The recorded and processed data are shown in Figure 4.

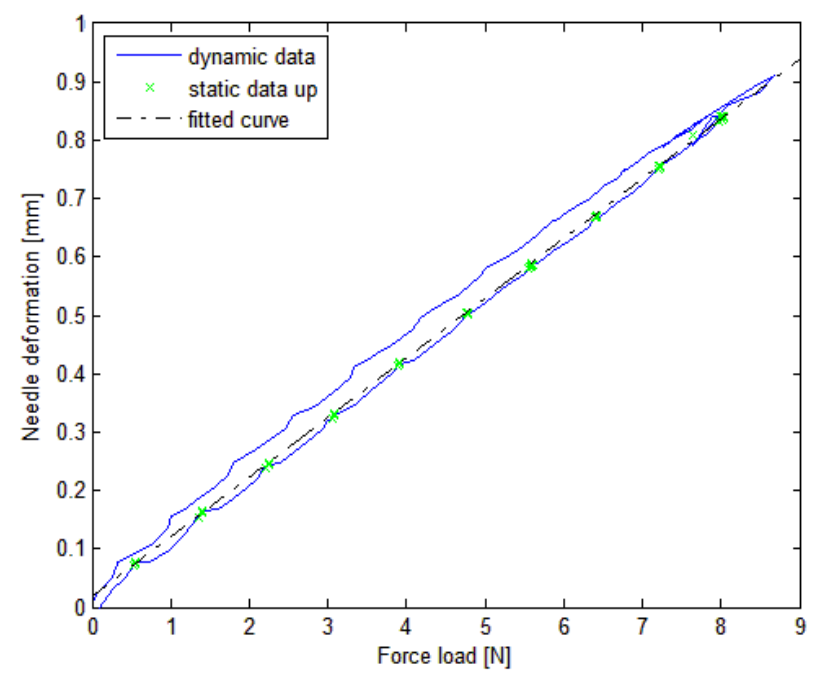

static set points, and also for the loading force's increases and decreases. The static data points show very good reproducibility (marked with green or 
blue crosses). The dynamic data - in between the static positions where needle deformation varies show always lower needle deformation under given force load compared to the static data points. It may be explained by the presence of friction forces and torques during needle motion, which decreases the bending needle reaction force. The static data show excellent linearity of $R=0.9999$ for the increase of the loading force. Unloading data points show distinguished hysteresis and higher needle deformation caused by the applied force.

We evaluated the needle Young modulus value using the static measured data under load increases. We measured inner and outer $D=0.617 \mathrm{~mm}$ and inner $d=0.392 \mathrm{~mm}$ needle diameter respectively and evaluated the second moment of area of the beam's cross-section by relation:

$$
I_{p}=\frac{\pi}{64}\left(D^{4}-d^{4}\right) \text {. }
$$

The Young modulus $E$ can be expresses from the elastic beam theory with equation:

$$
E=\frac{L^{3}}{48 I_{p}} \frac{F}{y},
$$

where $F$ is the loading force, $y$ is the beam deflection corresponding to the needle deformation and $L$ is the beam length. Our data show the needle Young modulus $E=234.8 \mathrm{GPa}$ with repeatability $\pm 0.5 \mathrm{GPa}$.

While the trueness of the measurement is mainly affected with correctness of the diameters measurement the total uncertainty of the measured Young modulus value $E=235 \pm 6 \mathrm{GPa}$ with $95 \%$ confidence bounds.

\section{b. Needle Two-Point Bending Test}

We prepared also a system for a needle 2-point bending test, as such load corresponds better to the expected load scheme of the eye endoscope, where the tube is fixed at the tool end and is loaded with a force by eye cornea structure. We fixed the needle to a 23G trocar and loaded it at a given distance from the port by the loading cell optical prism bevel edge.

The needle deformation response under 2-point bending test is shown in Figure 5.

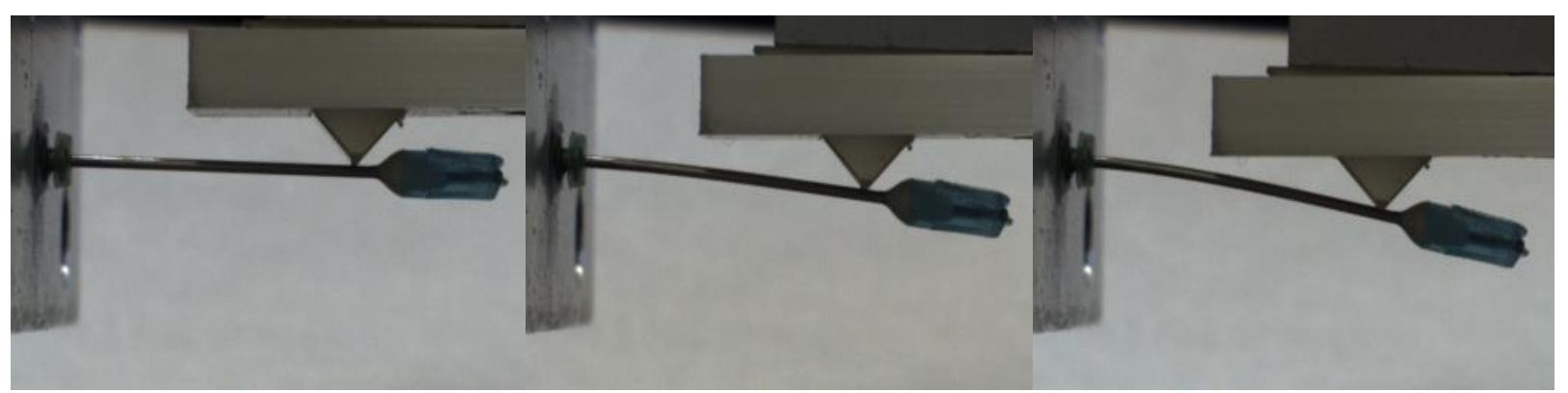

Figure 5: A detail view to the tested needle under 2-point bending load from zero (left image) to maximum used load (right image).

We kept the same measurement conditions for 2point bend test as for the previous 3-point bend test just with a changed value of the individual step distance set with the micrometer screw. As the needle has lower stiffness and higher deformation under the same load level, we performed loading data measurement in $250 \mu \mathrm{m}$ steps. The recorded and processed data are shown in Figure 6.

The measured data show similar characteristics as in the case of the 3-point bending test.

The static data show excellent linearity $R=$ 0.9998 for the increases of the loading force. Unloading data points show distinguished hysteresis of higher needle deformation to the applied force.

To the contrary to the 3-point bending test, the dynamic data give always higher needle deformation than for the static data load case. It may be caused by a different accumulation and release of the deformation energy of the needle during loading and unloading due to the different level and opposite direction of the friction force between the steel needle surface and glass loading prism in its contact point.

The correctness of this explanation needs to be proved with exact deformation profile measurement of the needle during its loading and unloading. 

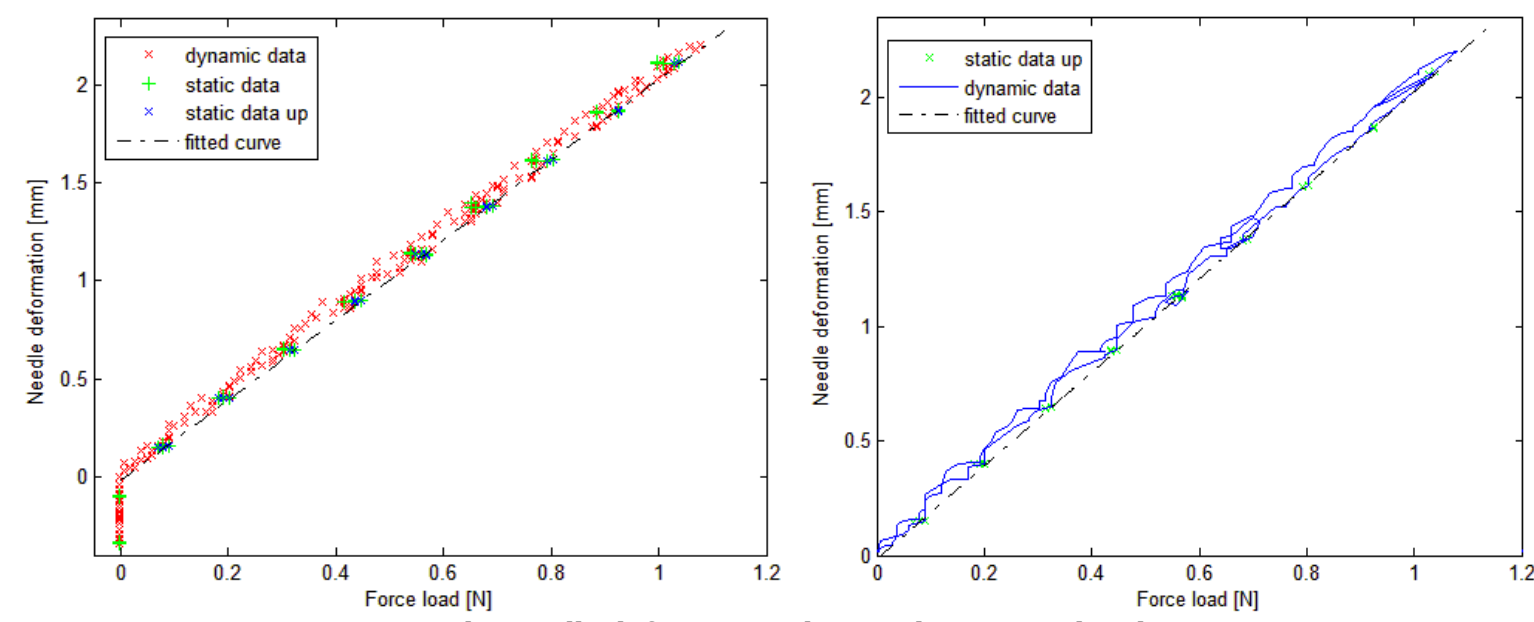

Figure 6: The needle deformation data under 2-point bending test.

\section{Conclusion}

The paper presented a new design of a simple loading system based on replacing the standard linear motion of the loading tip with approximate circular motion. We performed a mechanical analysis of the proposed system and proved a high reliability of about $10^{-4}$ within assumed working range. We performed the system calibration and load/deformation measurement in 3-point and 2point bending test of a needle specimen. Obtained data show excellent linearity for load increases. We use the 3-point bending test linear data for evaluation of the needle Young modulus. The value $E$ $=235 \pm 6 \mathrm{GPa}$ slightly higher than for the steel can be expresses by Young modulus increases by coldforming high strength to thin walls below $1 \mathrm{~mm}$ [5]. Notable hysteresis observed in the case of the specimen unloading needs to be correctly explained.

The system sensitivity was able to distinguish a discrepancy between linear characteristics of the static data measurement and dynamic data points. In the case of 3-point bending load we found that the needle deformation is always lower for the dynamic data points than for the static data points. The 2point bending load shows completely opposite behavior where the needle deformation is always higher for the dynamic data points than for the static data points.

We tried to explain the described data difference by different accumulation and release of the deformation energy of the needle during its loading and unloading due to the different level and opposite direction of the friction force between the steel needle surface and glass loading prism in its contact point. The correctness of this explanation needs to be proved with exact deformation profile measurement of the needle during its loading and unloading.

\section{References}

[1] Van de Berg, N. J., de Jong, T. L., van Gerwen, D. J., Dankelman, J., van den Dobbelsteen, J. J.: The influence of tip shape on bending force during needle insertion, Scientific Reports 7, 40477 (2017).

[2] Mahvash, M., Dupont, P. E.: Mechanics of Dynamic Needle Insertion into a Biological Material, In: IEEE TRANSACTIONS ON BIOMEDICAL ENGINEERING, 57(4), 934-943, (2010).

[3] Misra, S., Reed, K. B., Schafer, B. W., Ramesh, K. T., Okamura, A. M.: Mechanics of Flexible Needles Robotically Steered through Soft Tissue, The International Journal of Robotics Research 29(13) 1640-1660, (2010).

[4] Abbe, E.: Messapparate für Physiker (in German), Zeitschrift fur Instrumentenkunde, 10, 446-448, (1890).

[5] Mahendran, M.: The modulus of elasticity of steel - is it $200 \mathrm{GPa}$ ? In: Thirteenth International Specialty Conference on Cold-Formed Steel Structures, Missouri U.S.A., 641-648, (1996). 